\title{
Selection Criteria and Current Issues in Liver Transplantation for Hepatocellular Carcinoma
}

\author{
Toshimi Kaido \\ Division of Hepato-Biliary-Pancreatic and Transplant Surgery, Department of Surgery, Graduate \\ School of Medicine, Kyoto University, Kyoto, Japan
}

\section{Key Words}

Direct-acting antiviral agents · Expanded criteria · Hepatocellular carcinoma . Liver transplantation · Sarcopenia

\begin{abstract}
Liver transplantation (LT) is an ideal treatment for hepatocellular carcinoma (HCC) because it not only resects HCCs but it also replaces the underlying damaged liver with normal tissue. However, the selection criteria are still a matter of debate. After the introduction of the Milan criteria, some expanded criteria focusing on tumor size and number have been proposed. In addition, new expanded criteria considering tumor biology have been proposed using tumor markers and ${ }^{18} \mathrm{~F}$-fluorodeoxyglucose positron emission tomography. This review summarizes the selection criteria in LT for HCC and introduces current issues focusing on the treatment for hepatitis $C$ virus infection and the significance of sarcopenia in this field.
\end{abstract}

Copyright @ 2016 S. Karger AG, Basel

\section{Background}

Liver transplantation (LT) is an ideal treatment for hepatocellular carcinoma (HCC) because it not only resects the disease but it also replaces the underlying damaged liver with normal tissue. In other words, LT is a radical treatment for HCC in that it can simultaneously treat intrahepatic metastasis as well as multicentric carcinogenesis. However, the selection criteria are still a matter of debate $[1,2]$. This review summarizes the selection criteria and current issues in LT for HCC while at the same time introducing new data. 


\section{Selection Criteria in LT for HCC}

In Western countries, with the advent of the Milan criteria [3], deceased donor LT (DDLT) for HCC has achieved favorable survival rates, comparable to those for nonmalignant liver diseases. In contrast, the high incidence of HCC due to the high endemic rate of hepatitis $\mathrm{B}$ or $\mathrm{C}$ virus (HBV/HCV) and the critical shortage of deceased donor organs have led to the rapid development of living donor LT (LDLT) in Eastern countries including Japan, Korea, and China. In Japan, there is minimal chance for patients with HCC to undergo DDLT due to the extreme shortage of deceased donor livers. Therefore, the category of LT and the concept of selection criteria of LT for HCC are different between Western and Eastern countries.

After the introduction of the Milan criteria, some expanded criteria focusing on tumor size and number have been proposed, because the aforementioned criteria are considered too restrictive [4-6]. Moreover, imaging modalities have developed dramatically in the last two decades. The imaging modality used in the establishment of the Milan criteria was computed tomography (CT) during arterial portography (CTAP). Currently, small HCCs can be easily and accurately diagnosed by multidetector CT and gadolinium ethoxybenzyl diethylenetriamine pentaacetic acid (Gd-EOB-DTPA)-enhanced magnetic resonance imaging (MRI) without using CTAP. Therefore, a larger number of tumors can be detected at present compared with the number in the Milan era. Yao et al. reported that, in 168 HCC patients who met the University of California San Francisco (UCSF) criteria (a single lesion $\leq 6.5 \mathrm{~cm}$ in diameter or 2-3 lesions $\leq 4.5 \mathrm{~cm}$ with a total diameter $\leq 8 \mathrm{~cm}$ on the basis of preoperative radiological data), the 5-year recurrence-free probability and recurrence-free survival rates were $90.9 \%$ and $80.7 \%$, respectively [4]. Sugawara et al. reported that 72 HCC patients within their 5-5 rule (up to five nodules with a maximum diameter of $5 \mathrm{~cm}$ ) achieved a 3-year recurrencefree survival rate of $94 \%$ after LDLT [5]. Mazzaferro et al. proposed more liberal criteria than the Milan criteria: the "up-to-7 criteria" (HCC with seven as the sum of the size of the largest tumor in $\mathrm{cm}$ and the number of tumors) [6]. They showed that patients with HCCs beyond the Milan criteria, but within the "up-to-7 criteria" in the absence of microvascular invasion $(\mathrm{n}=283)$, had a similar survival rate compared with patients within the Milan criteria irrespective of microvascular invasion $(n=444)$.

In contrast to DDLT, the indications for LDLT for HCC are decided based on the balance between risks to the live donor and benefits to the recipient. As a result, many Asian transplantation centers also have adopted expanded criteria beyond the standard criteria of Milan or UCSF. In general, however, the more the morphological limits of the selection criteria expand, the more the recurrence rates after LT increase. On the basis of the idea that incorporating tumor biological markers and predicting microvascular invasion and poor differentiation can exclude patients with a high risk of recurrence before LT, some expanded criteria that include such markers have recently been proposed [7-9]. Toso et al. proposed new selection criteria by combining total tumor volume $\leq 115 \mathrm{~cm}^{3}$ and $\alpha$-fetoprotein (AFP) $\leq 400 \mathrm{ng} / \mathrm{ml}$ [7]. Zheng et al. also proposed expanded selection criteria, the Hangzhou criteria, incorporating the AFP level in cases with a total tumor diameter $>8 \mathrm{~cm}$ [8].

On the other hand, optimal selection criteria should be established based on target outcomes after implementation of the criteria. The Kyoto group set target outcomes as a 5-year survival rate $\geq 80 \%$ and a 5 -year recurrence rate $\leq 10 \%$. Based on these target outcomes, the Kyoto group also established new expanded selection criteria, the Kyoto criteria, by combining three independent significant risk factors for recurrence: tumor number and tumor size based on the findings of pretransplant imaging and tumor markers; tumor number $\leq 10$, maximal diameter of each tumor $\leq 5 \mathrm{~cm}$; and serum des-gamma-carboxy prothrombin (DCP) levels $\leq 400 \mathrm{mAU} / \mathrm{ml}$ [9]. DCP, also known as protein induced by vitamin $\mathrm{K}$ absence or antagonist II (PIVKA-II), is a well-known tumor marker of HCC whose expression is significantly 
correlated with poor prognosis $[10,11]$. In a retrospective analysis, 78 patients who met the Kyoto criteria showed significantly lower 5 -year recurrence rates $(4.9 \%)$ than the 40 patients who exceeded them $(60.5 \%$, p <0.001) [12]. Similarly, 5-year survival rates in patients within the Kyoto criteria (86.7\%) were significantly higher compared with patients exceeding the criteria $(34.4 \%, \mathrm{p}<0.001)$.

Recently, Kaido et al. prospectively validated the utility of the Kyoto criteria [13]. Intention-to-treat analysis of 62 patients who underwent LDLT after implementation of the Kyoto criteria showed that the 5-year overall survival rate and the recurrence rate were $82 \%$ and $7 \%$, respectively. These results satisfy our target outcomes.

Regarding tumor biology, the incidence of microvascular invasion and poorly differentiated HCCs were compared between patients within and exceeding the Kyoto, Milan, and UCSF criteria, respectively [13]. The incidence of microvascular invasion was significantly lower in patients within the Kyoto criteria than in patients exceeding the Kyoto criteria $(p<0.001)$. The incidence of poorly differentiated HCCs was also significantly lower in patients within the Kyoto criteria than in patients exceeding the Kyoto criteria $(\mathrm{p}=0.010)$. In contrast, the incidence of poorly differentiated HCCs did not differ significantly between patients within and exceeding the Milan or the UCSF criteria, while the incidence of microvascular invasion was significantly lower in patients within these criteria than in patients exceeding these criteria. These findings indicate that incorporating tumor markers into the selection criteria can successfully widen the indication without increasing the recurrence rate.

As an alternative method of predicting tumor biology, some investigators have reported the usefulness of ${ }^{18} \mathrm{~F}$-fluorodeoxyglucose positron emission tomography for selecting appropriate candidates for LT for HCC $[14,15]$. Kornberg et al. showed that negative PET findings (odds ratio $=21.6, \mathrm{p}<0.001$ ), AFP level $<400 \mathrm{IU} / \mathrm{mL}$ (odds ratio=3.3, $\mathrm{p}=0.013$ ), and total tumor diameter $<10 \mathrm{~cm}$ (odds ratio $=3.0, \mathrm{p}=0.022$ ) were identified as pretransplant prognostic variables for recurrence-free survival [14]. Moreover, a PET-positive status was revealed as the only independent clinical predictor of tumor-related patient dropout from the waiting list (hazard ratio $=5.7, \mathrm{p}=0.01$ ). Lee et al. reported that the 1- and 2-year recurrence-free survival rates were $97 \%$ versus $57 \%$ and $97 \%$ versus $42 \%$ for PET-negative patients versus PET-positive patients, respectively [15]. In a multivariate analysis, PET-positivity and the presence of microvascular invasion were the only predictors of tumor recurrence. Lee et al. most recently reported that patients with HCC beyond the Milan criteria with a PET-negative status and a total tumor size $<10 \mathrm{~cm}$ showed similar overall survival and disease-free survival compared with patients with HCC within the Milan criteria [16].

Conversely, Dubay et al. reported the usefulness of pretransplant liver biopsy and proposed that LT for advanced-moderate to well-differentiated HCC can be performed safely with excellent 5-year overall and disease-free survival in the absence of HCC size and tumor number restrictions [17]. However, biopsy for patients with decompensated cirrhotic liver is not always possible due to the retention of ascites and the fact that a biopsy itself holds a risk of bleeding as well as tumor dissemination. Moreover, a biopsy cannot accurately diagnose the most advanced degree of differentiation due to the heterogeneity of HCC tumors. Therefore, noninvasive methods including tumor markers and PET are desirable for predicting tumor biology. In Japan, a working group is now developing new expanded selection criteria by incorporating tumor size, number, and tumor markers using a nationwide database. 


\section{Current Issues in LT for HCC}

\section{Antiviral Treatment after LT}

HBV or HCV infection is the leading cause of HCC, especially in East Asia. Therefore, antiviral treatment for grafts is a crucial issue in prolonging graft survival. Regarding HBV infection, posttransplant administration of anti-hepatitis B surface immunoglobulin and reverse transcriptase inhibitors can nearly always prevent HBV reinfection of grafts. In contrast, almost all grafts develop recurrent HCV infection, and 15-30\% of grafts develop liver cirrhosis. There are three types of treatment for HCV reinfection after LT: pretransplant therapy, preemptive therapy, and on-demand therapy.

Pretransplant therapy is a favorable option for well-compensated patients with HCC or mildly decompensated liver cirrhosis, since up to two-thirds of patients who become HCV ribonucleic acid-negative on treatment are suggested to be HCV infection-free after LT [18]. The rates of on-treatment sustained virological response (SVR) were 30\% (range, 18-56\%) in genotype 1 and $83 \%$ (range, $82-100 \%$ ) in genotype $2 / 3$ recipients, as found in an analysis of 275 patients who underwent pretransplant antiviral therapy with mostly mild to moderate liver decompensation, receiving either a LDLT or a DDLT $[18,19]$.

Preemptive antiviral therapy that is started within weeks of the transplantation is limited by tolerability. Rates of SVR vary from 8-39\% [18, 20]. Posttransplant antiviral therapy initiated upon histological evidence of recurrence is therefore the mainstay of treatment. The Kyoto group reported that a combination of pegylated interferon alpha- $2 \mathrm{~b}$ and ribavirin achieved a 50\% SVR rate for recurrent hepatitis C genotype $1 \mathrm{~b}$ [21]. However, these results are still unsatisfactory, since about half of the recipients with HCV reinfection develop liver cirrhosis, and, if uncontrolled, require re-LT.

Recently, management of hepatitis $\mathrm{C}$ has dramatically changed with the introduction of direct-acting antiviral agents (DAAs). Kumada et al. reported that 24-weeks of treatment with daclatasvir and asunaprevir could achieve a high rate of SVR in patients with HCV genotype $1 \mathrm{~b}$ who were ineligible, intolerant, or had not responded to prior interferon-based therapy with a low incidence of severe adverse events [22]. Accordingly, anti-HCV therapy can now be safely administered to patients after LT without using interferon. Moreover, the drug-drug interaction between calcineurin inhibitors and the new DAAs is weaker compared with the first and second generation DAAs. Our group most recently reported the usefulness of interferon-free therapy with DAAs in patients with progressive severe cholestatic hepatitis $\mathrm{C}$ with acute cellular rejection and bacterial infection after LT [23]. Therefore, HCV recurrence after LT for HCC might not be a serious problem in the near future.

\section{Expansion of Donor Pool}

Needless to say, DDLT is preferable to LDLT in terms of donor safety, even in patients with HCC. However, the number of deceased donors is still limited and is in short supply globally. Most recently, Croome et al. reported that there was no difference in recurrencefree survival $(p=0.29)$ or cumulative incidence of HCC recurrence $(p=0.91)$ between patients who underwent LT with a donation made after brain death $(n=340)$ or a donation made after cardiac death $(n=57)$ [24]. In circumstances where the number of deceased donors is limited, besides using donation allografts after cardiac death, optimizing the use of marginal donor grafts through various innovations, including development of organ preservation methods, is necessary to increase the number of available allografts.

\section{Significance of Sarcopenia}

Sarcopenia is defined as a syndrome characterized by progressive and generalized loss of skeletal muscle mass and strength [25]. The prognostic significance of sarcopenia has 

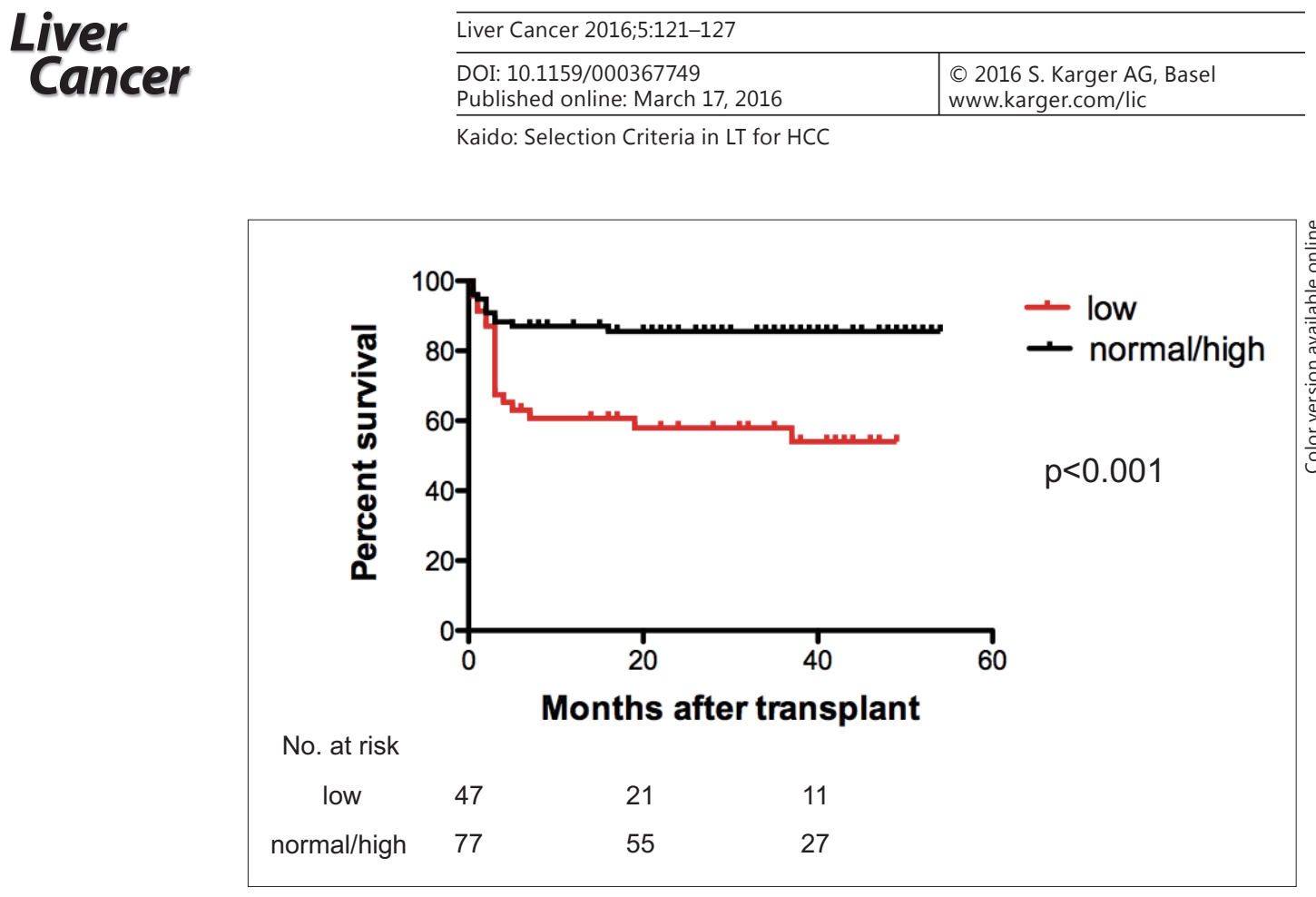

Fig. 1. Overall survival rates according to skeletal muscle mass in patients undergoing LDLT.

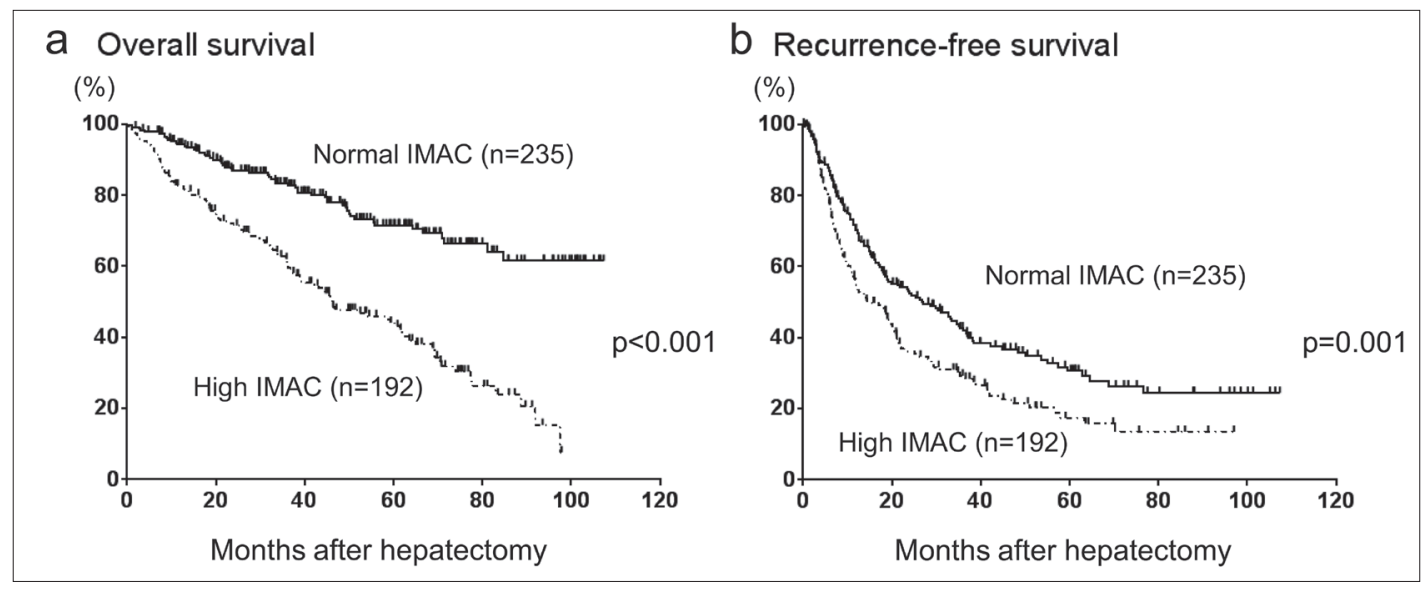

Fig. 2. Overall survival rates (a) and recurrence-free survival rates (b) after hepatectomy for HCC according to preoperative IMAC.

been reported in various kinds of diseases including cancer [26, 27]. In patients undergoing LDLT, we showed that the overall survival rate in patients with preoperative low skeletal muscle mass was significantly lower than in patients with normal/high skeletal muscle mass ( $p<0.001$ ) (fig. 1) [28]. Moreover, we recently focused on muscle quality evaluated by intramuscular adipose tissue content (IMAC) as a novel sarcopenic factor. The overall survival rate after LDLT was significantly lower in patients with high IMAC (= low muscle quality) than in patients with normal/low IMAC $(=$ normal muscle quality) $(\mathrm{p}<0.001)$ [29]. In addition, in patients undergoing hepatectomy for HCC, the overall and recurrence-free survival rates were significantly lower in patients with high IMAC values than in patients with normal IMAC values ( $p<0.001, p=0.001$, respectively; fig. 2a, b) [30]. On multivariate analysis, preoperative low quality of skeletal muscle evaluated by IMAC was an independent risk factor for poor survival in patients undergoing LDLT and in patients undergoing hepatectomy for HCC $[29,30]$. The mechanisms by which low quality of skeletal muscle accompanies an increased risk of mortal- 
ity and morbidity are not fully understood. Recent evidence has shown that skeletal muscle loss with increasing adipose tissue leads to the synthesis and secretion of various kinds of proinflammatory adipokines such as leptin, tumor necrosis factor alpha, interleukin (IL)-1, and IL-6 [31]. Lutz et al. [32] showed that an increase in such adipokines and a decrease in adiponectin or myokines, including IL-15 levels, in the sarcopenic population had inhibitory effects on the immune system, especially with respect to natural killer lymphocytes in innate immunity. Sarcopenia therefore appears to play a crucial role in the outcomes of treatment for HCC.

Taken together, aggressive perioperative nutritional therapy with rehabilitation could be a new strategy to ameliorate outcomes for patients who undergo LT for HCC.

\section{Key Messages}

Compared with the Milan criteria, expanded selection criteria that consider tumor biology can efficiently exclude patients at high risk for postoperative recurrence, and more HCC patients can be candidates for LT with acceptable outcomes. Aggressive perioperative nutritional therapy together with rehabilitation could be a new strategy to ameliorate outcomes for patients who undergo LT for HCC.

\section{Disclosure Statement}

The author declares no conflict of interest.

\section{References}

1 Chan SC: Liver transplantation for hepatocellular carcinoma. Liver Cancer 2013;2:338-344.

2 Akamatsu N, Sugawara Y, Kokudo N: Living donor liver transplantation for patients with hepatocellular carcinoma. Liver Cancer 2014;3:108-118.

3 Mazzaferro V, Regalia E, Doci R, Andreola S, Pulvirenti A, Bozzetti F, Montalto F, Ammatuna M, Morabito A, Gennari L: Liver transplantation for the treatment of small hepatocellular carcinomas in patients with cirrhosis. N Engl J Med 1996;334:693-699.

4 Yao FY, Xiao L, Bass NM, Kerlan R, Ascher NL, Roberts JP: Liver transplantation for hepatocellular carcinoma: validation of the UCSF-expanded criteria based on preoperative imaging. Am J Transplant 2007;7:2587-2596.

5 Sugawara Y, Tamura S, Makuuchi M: Living donor liver transplantation for hepatocellular carcinoma: Tokyo University series. Dig Dis 2007;25:310-312.

6 Mazzaferro V, Llovet JM, Miceli R, Bhoori S, Schiavo M, Mariani L, Camerini T, Roayaie S, Schwartz ME, Grazi GL, Adam R, Neuhaus P, Salizzoni M, Bruix J, Forner A, De Carlis L, Cillo U, Burroughs AK, Troisi R, Rossi M, Gerunda GE, Lerut J, Belghiti J, Boin I, Gugenheim J, Rochling F, Van Hoek B, Majno P, Metroticket Investigator Study Group: Predicting survival after liver transplantation in patients with hepatocellular carcinoma beyond the Milan criteria: a retrospective, exploratory analysis. Lancet Oncol 2009;10:35-43.

7 Toso C, Asthana S, Bigam DL, Shapiro AM, Kneteman NM: Reassessing selection criteria prior to liver transplantation for hepatocellular carcinoma utilizing the Scientific Registry of Transplant Recipients database. Hepatology 2009;49:832-838.

8 Zheng SS, Xu X, Wu J, Chen J, Wang WL, Zhang M, Liang TB, Wu LM: Liver transplantation for hepatocellular carcinoma: Hangzhou experiences. Transplantation 2008;85:1726-1732.

9 Takada Y, Ito T, Ueda M, Sakamoto S, Haga H, Maetani Y, Ogawa K, Ogura Y, Oike F, Egawa H, Uemoto S: Living donor liver transplantation for patients with HCC exceeding the Milan criteria: a proposal of expanded criteria. Dig Dis 2007;25:299-302.

10 Yamamoto K, Imamura H, Matsuyama Y, Hasegawa K, Beck Y, Sugawara Y, Makuuchi M, Kokudo N: Significance of alpha-fetoprotein and des-gamma-carboxy prothrombin in patients with hepatocellular carcinoma undergoing hepatectomy. Ann Surg Oncol 2009;16:2795-2804.

11 Miyaaki H, Nakashima O, Kurogi M, Eguchi K, Kojiro M: Lens culinaris agglutinin-reactive alpha-fetoprotein and protein induced by vitamin $\mathrm{K}$ absence II are potential indicators of a poor prognosis: a histopathological study of surgically resected hepatocellular carcinoma. J Gastroenterol 2007;42:962-968. 
12 Ito T, Takada Y, Ueda M, Haga H, Maetani Y, Oike F, Ogawa K, Sakamoto S, Ogura Y, Egawa H, Tanaka K, Uemoto S: Expansion of selection criteria for patients with hepatocellular carcinoma in living donor liver transplantation. Liver Transpl 2007;13:1637-1644.

13 Kaido T, Ogawa K, Mori A, Fujimoto Y, Ito T, Tomiyama K, Takada Y, Uemoto S: Usefulness of the Kyoto criteria as expanded selection criteria for liver transplantation for hepatocellular carcinoma. Surgery 2013;154:1053-1060.

14 Kornberg A, Küpper B, Tannapfel A, Büchler P, Krause B, Witt U, Gottschild D, Friess H: Patients with non[18 F]fludeoxyglucose-avid advanced hepatocellular carcinoma on clinical staging may achieve long-term recurrence-free survival after liver transplantation. Liver Transpl 2012;18:53-61.

15 Lee JW, Paeng JC, Kang KW, Kwon HW, Suh KS, Chung JK, Lee MC, Lee DS: Prediction of tumor recurrence by 18F-FDG PET in liver transplantation for hepatocellular carcinoma. J Nucl Med 2009;50:682-687.

16 Lee SD, Kim SH, Kim SK, Kim YK, Park SJ: Clinical Impact of 18F-Fluorodeoxyglucose Positron Emission Tomography/Computed Tomography in Living Donor Liver Transplantation for Advanced Hepatocellular Carcinoma. Transplantation 2015; [Epub ahead of print].

17 DuBay D, Sandroussi C, Sandhu L, Cleary S, Guba M, Cattral MS, McGilvray I, Ghanekar A, Selzner M, Greig PD, Grant DR: Liver transplantation for advanced hepatocellular carcinoma using poor tumor differentiation on biopsy as an exclusion criterion. Ann Surg 2011;253:166-172.

18 Terrault NA: Hepatitis C therapy before and after liver transplantation. Liver Transpl 2008;14(Suppl 2):S58-S66.

19 Terrault NA, Shiffman ML, Lok AS, Saab S, Tong L, Brown RS Jr, Everson GT, Reddy KR, Fair JH, Kulik LM, Pruett TL, Seeff LB, A2ALL Study Group: Outcomes in hepatitis C virus-infected recipients of living donor vs. deceased donor liver transplantation. Liver Transpl 2007;13:122-129.

20 Sugawara Y, Makuuchi M, Matsui Y, Kishi Y, Akamatsu N, Kaneko J, Kokudo N: Preemptive therapy for hepatitis C virus after living-donor liver transplantation. Transplantation 2004;78:1308-1311.

21 Ueda Y, Takada Y, Marusawa H, Egawa H, Uemoto S, Chiba T: Individualized extension of pegylated interferon plus ribavirin therapy for recurrent hepatitis $\mathrm{C}$ genoty pe $1 \mathrm{~b}$ after living-donor liver transplantation. Transplantation 2010;90:661-665.

22 Kumada H, Suzuki Y, Ikeda K, Toyota J, Karino Y, Chayama K, Kawakami Y, Ido A, Yamamoto K, Takaguchi K, Izumi N, Koike K, Takehara T, Kawada N, Sata M, Miyagoshi H, Eley T, McPhee F, Damokosh A, Ishikawa H, Hughes E: Daclatasvir plus asunaprevir for chronic HCV genotype 1b infection. Hepatology 2014;59:20832091.

23 Ueda Y, Kaido T, Hatano E, Ohtsuru S, Uemoto S: Safe and effective treatment with daclatasvir and asunaprevir in a liver transplant recipient with severe cholestatic hepatitis C. Hepatol Res 2015; [Epub ahead of print].

24 Croome KP, Lee DD, Burns JM, Musto K, Paz D, Nguyen JH, Perry DK, Harnois DM, Taner CB: The Use of Donation After Cardiac Death Allografts Does Not Increase Recurrence of Hepatocellular Carcinoma. Am J Transplant 2015; [Epub ahead of print].

25 Cruz-Jentoft AJ, Baeyens JP, Bauer JM, Boirie Y, Cederholm T, Landi F, Martin FC, Michel JP, Rolland Y, Schneider SM, Topinková E, Vandewoude M, Zamboni M, European Working Group on Sarcopenia in Older People: Sarcopenia: European consensus on definition and diagnosis: Report of the European Working Group on Sarcopenia in Older People. Age Ageing 2010;39:412-423.

26 Prado CM, Lieffers JR, McCargar LJ, Reiman T, Sawyer MB, Martin L, Baracos VE: Prevalence and clinical implications of sarcopenic obesity in patients with solid tumours of the respiratory and gastrointestinal tracts: a population-based study. Lancet Oncol 2008;9:629-635.

27 Tan BH, Birdsell LA, Martin L, Baracos VE, Fearon KC: Sarcopenia in an overweight or obese patient is an adverse prognostic factor in pancreatic cancer. Clin Cancer Res 2009;15:6973-6979.

28 Kaido T, Ogawa K, Fujimoto Y, Ogura Y, Hata K, Ito T, Tomiyama K, Yagi S, Mori A, Uemoto S: Impact of sarcopenia on survival in patients undergoing living donor liver transplantation. Am J Transplant 2013;13:15491556.

29 Hamaguchi Y, Kaido T, Okumura S, Fujimoto Y, Ogawa K, Mori A, Hammad A, Tamai Y, Inagaki N, Uemoto S: Impact of quality as well as quantity of skeletal muscle on outcomes after liver transplantation. Liver Transpl 2014;20:1413-1419.

30 Hamaguchi Y, Kaido T, Okumura S, Ito T, Fujimoto Y, Ogawa K, Mori A, Hammad A, Hatano E, Uemoto S: Preoperative intramuscular adipose tissue content is a novel prognostic predictor after hepatectomy for hepatocellular carcinoma. J Hepatobiliary Pancreat Sci 2015;22:475-485.

31 Tilg H, Moschen AR: Adipocytokines: mediators linking adipose tissue, inflammation and immunity. Nat Rev Immunol 2006;6:772-783.

32 Lutz CT, Quinn LS: Sarcopenia, obesity, and natural killer cell immune senescence in aging: altered cytokine levels as a common mechanism. Aging (Albany, NY Online) 2012;4:535-546. 